Apidologie, 1971, 2 (3), 249-257.

\title{
ÜBER DEN EINFLUSS DER KONSERVIERUNGSMETHODE AUF STERILITÄT UND MOTILITÄT DER BIENENSPERMIEN (APIS MELLIFICA L.)
}

Influence de la méthode de conservation sur la stérilité et la motilité des spermatozoüdes d'abeille (Apis mellifica L).

\author{
Marie MACHOVÁ und Vladimír VESELÝ \\ Bienenforschungsinstitut in Dol bei Libčice, ČSSR
}

\begin{abstract}
SUMMARY
THE INFLUENCE OF PRESERVATION METHODS ON STERILITY

AND MOTILITY OF THE SPERMS OF APIS MELLIFICA L.
\end{abstract}

1. A secondary infection of the originally sterile sperm may occur while being collected from the drone's penis, at the preservation or when opening the preserves.

2. The bacteria in infected sperm were identified as Streptococcus epidermis, Bacillus brevis, Gaffkya tetragena and Citrobacter (Escherichia) freundii. All identified bacteria belong to the banal microflora. In no case they were pathogenic for bees or man. The most frequent source of infection was the human epidermis.

3. Sterility of the preserves can be guaranteed by a careful collection of the sperm or by addition of Lysozyme or Chloramphenicol solutions in a concentration of $1 \mathrm{mg} / \mathrm{ml}$ at a ratio of $1: 10$.

4. Sterility is protecting the preserved sperm from a premature decay but does not lengthen the maximum time of storage ( 60 days).

5. At the application of Chloramphenicol, hitherto unknown side effects were observed which distinctly increased the motility of the sperm after 60 days of storage.

6. Further examination should handle with these side effects of Chloramphenicol or other substances interfering with the spermic metabolism. 


\section{ZUSAMMENFASSUNG}

1. Das ursprünglich sterile Sperma wird sekundär bei der Abnahme vom Begattungsorgan des Drohns, bei der Herstellung oder beim Öffnen der Konserven infiziert.

2. Die Bakterien im infizierten Sperma wurden als Streptococcus epidermidis, Bacillus brevis, Gaffkya tetragena und Citrobacter (Escherichia) freundii identifiziert. Alle gefundenen Arten gehören zur banalen Mikroflora, in keinem einzigen Falle handelte es sich um bienenoder menschenpathogene Formen. Häufigste Infektionsquelle war die Menschenhaut.

3. Sterilität der Konserven kann man durch eine sorgfältige Abnahme des Spermas oder durch Zusatz von Lysosym- oder Chloramphenikollösung in der Konzentration $1 \mathrm{mg} / \mathrm{ml}$ im Verhältnis 1 : 10 zum Sperma sicherstellen.

4. Sterilität schützt das konservierte Sperma vor vorzeitiger Entwertung, verlängert aber nicht die maximale Lagerungsfrist (60 Tage).

5. Beim Chloramphenikol wurden bisher unbekannte Nebenwirkungen entdeckt, die die Motilität der 60 Tage gelagerten Spermien deutlich erhöhen.

6. Weitere Forschungen sollten sich auf diese Nebenwirkungen von Chloramphenikol oder von anderen in den Spermienmetabolismus eingreifenden Stoffen richten.

Obwohl die Bienenspermien in vivo, nämlich im Receptaculum der Königin, mehrere Jahre am Leben bleiben, ist es bisher nicht gelungen, die Konservierung des Spermas in vitro befriedigend zu lösen.

Verhältnismässig gute Erfolge erzielten TABER und BLUm (1960) und TABER (1961) durch Aufbewahrung des Spermas in zugeschmolzenen Kapillaren bei Dunkelheit und Zimmertemperatur. TABER und BLUM (1960) führten noch mit 68 Tage aufbewahrten Spermien teilweise erfolgreiche Besamungen durch. Von den 4 mit diesem Sperma besamten Königinnen legte 1 Arbeiterinneneier, 1 Drohneneier; 2 Königinnen gingen ein. Dem konservierten Sperma wurde Tetracyclin in nicht angegebener Menge zugesetzt.

Nach Zusatz von Streptomycin und Tetracyclin zum konservierten Sperma erzielten Poole une TABer (1969) noch nach 16-18 Wochen Lagerung vereinzelt erfolgreiche Besamungen. Nach 23-25 Wochen zeigte $1 \%$ der Spermien noch normale Beweglichkeit, der Rest war unbeweglich.

Škrobal, Veselý und HrdÝ (1966) stellten fest, daß die Zahl der in die Samenblase eingedrungenen Spermien bei dem ohne Zugabe von Schutzmitteln konservierten Sperma niedriger ist als bei frischem Sperma. Von $1 \mathrm{~mm}^{3}$ 7-34. Tage aufbewahrtem Sperma sind in die Samenblasen der inseminierten Königinnen durchschnittlich nur 155000 (maximal 484 000) eingedrungen, von jedem $\mathrm{mm}^{3}$ des frischen Spermas dagegen durchschnittlich 626000 (maximal 1114000$)$ Spermien.

Taber und Blum (1960) und ŠKrobal, Veselý und HrdÝ (1966) fanden bei manchen Spermakonserven deutliche Anzeichen von Infektionen, die durch Änderung der Farbe, durch Zerreissen der Spermasäule in der Kapillare, durch höhere Viskosität des Inhalts und durch üblen Geruch beim Öffnen der Konserven bemerkbar wurden. Sie sehen in bakteriellen Infektionen eine der möglichen Ursachen für die kurze Lebensdauer der konservierten Spermien. 
Ziel der vorliegenden Arbeit ist es deshalb, festzustellen, wie weit man durch garantierte Sterilität des Spermas in der Konserve die Motilität und damit die Brauchbarkeit der Spermien beeinflussen kann. Wir wollten ferner das eventuelle Vorkommen von Mikroorganismen primärer oder sekundärer Herkunft und die Zusammensetzung der Mikroflora in den Spermakonserven feststellen. Schließlich wollten wir klären, ob eine sterile Abnahme von Sperma möglich ist und welche Ergebnisse man von Schutzstoffen erwarten kann.

\section{MATERIAL UND METHODIK}

\section{Sperma}

Reines Sperma ohne Schleimbeimischung saugten wir von den ausgestülpten Begattungsorganen von mehr als 12 Tage alten Drohnen, die von begatteten und unbegatteten Königinnen der Rasse Apis mellifica carnica Pollm. stammten, in die Spritzennadel des Inseminationsapparates ein (VEselÝ, 1961). Die Ausstülpung erreichten wir durch Betäubung der Drohnen mit Chloroformdämpfen, die Ejakulation durch Druck auf den Hinterleib.

Die Spermakonserven stellten wir nach der Methodik von TABER (1961) her. Die spermasäule in der Kapillare fügten wir durch 1-2 minütiges Zentrifugieren bei 6000 Umdrehungen zusammen. Der Inhalt der einzelnen Konserven betrug 8-12 $\mathrm{mm}^{3}$ Sperma.

\section{Stoffe zum Schutz des Spermas gegen bakterielle Infektionen}

1. Lysosym, ein Enzym, das in den Zellwänden von Mikroben gewisse Mucopolysaccharide spaltet (STÁRKA, 1959). Wir haben es wegen seiner bakteriziden Wirkung auf eine ganze Reihe apathogener oder wenig pathogener, auf der menschlichen Haut und auf Schleimhäuten häufig vorkommender Mikroben gewählt (SEDLÁk und Kol., 1962). Wir benutzten das von der Firma Zdravotnické zásobobání, Heilmittelfabrik, hergestellte Lysosym. Von der konzentrierten Lysosymlösung, die mit Hilfe eines Seitz-Filters EK sterilisiert wurde, haben wir Lösungen mit den Konzentrationen $10 \mathrm{mg} / \mathrm{ml}, 5 \mathrm{mg} / \mathrm{ml}$ und $1 \mathrm{mg} / \mathrm{ml}$ vorbereitet.

2. Chloramphenikol, ein Antibioticum mit einem breiten antimikrobiellen Spektrum. Es wirkt auf gramnegative und grampositive Bakterien und Kokken, Spirochäten, Actinomyceten, Rickettsien und auf manche grosse Viren. Auf die meisten Mikroben wirkt es bakteriostatisch schon bei Konzentrationen von $0,001-0,01 \mathrm{mg} / \mathrm{ml}$ (Modr, HeřmanskÝ und VlčEK, 1969). Von der konzentrierten Chloramphenikollösung (Erzeugnis der Firma Zdravotnické zásobování, Heilmittelfabrik) haben wir eine Lösung mit der Konzentration $1 \mathrm{mg} / \mathrm{ml}$ vorbereitet.

Lösungen der Schutzstoffe setzten wir bei der Spermaabnahme durchweg im Verhältnis 1 : 10 zum Sperma zu.

\section{Öffnen der Konserven}

Die Ober fläche der Kapillaren haben wir nach Entfernung des Etiketts mit $96 \%$ igem Äthylalkohol gereinigt. Dann schnitten wir beide Enden der Kapillare ab und preßten den Inhalt mit einer ausgeglühten Nadel heraus.

\section{Bewertung der Motilität}

Den Inhalt der Konserve (bis auf einen winzigen, zur bakteriologischen Analyse dienenden Rest) verrührten wir im hängenden Tropfen mit $0.04 \mathrm{ml}$ einer $1 \%$ igen NaCl-Lösung. Dann beobachteten wir bei Zimmertemperatur die Spermienbewegung mikroskopisch bei 200 facher Vergrösserung bis zum Austrocknen des Tropfens (etwa 1 Stunde). Während dieser Zeit durchlüfteten wir den Tropfen ab und zu durch Mischen.

Wir bewerteten die maximale Motilität der Spermien, die bis zur Erreichung der maximalen Motilität vergehende Zeitspanne und die Dauer der Spermienbewegung. Die Motilität haben wir in die Bewertungsstufen 0-6 eingeteilt. Stufe 0 bedeutet : Spermien ohne jede Bewegung und Struktur; Stufe 1 : Spermien ohne sichtbare Bewegung, sie bilden aber am 


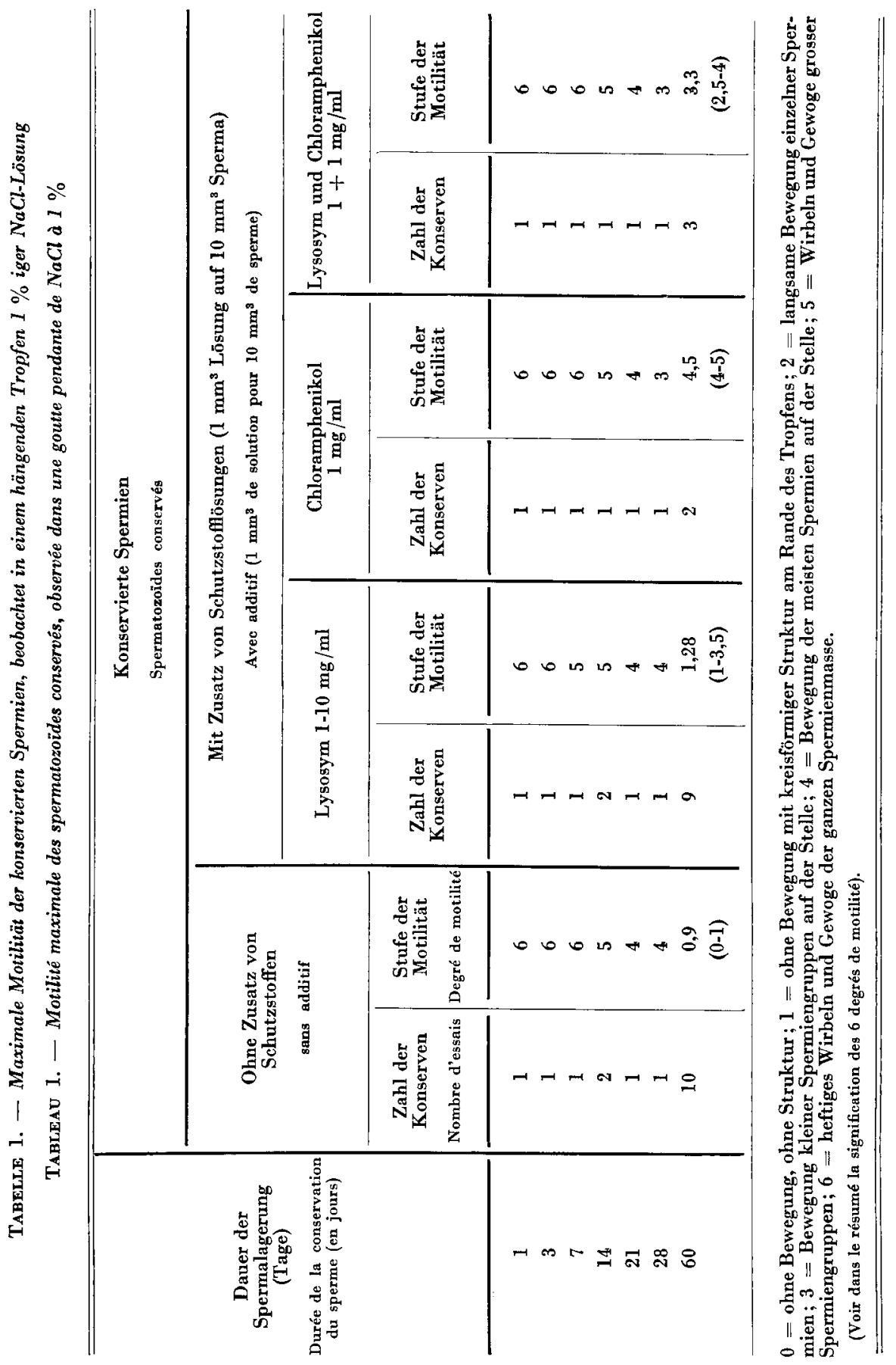


Rande des Tropfens eine kreisförmige Struktur; Stufe 2 : langsame Bewegung einzelner Spermien; Stufe 3 : Bewegung kleiner Spermiengruppen auf der Stelle; Stufe 4:Bewegung der meisten Spermien auf der Stelle; Stufe 5 : Wirbeln und Gewoge grosser Spermiengruppen; Stufe 6 : heftiges Wirbeln und Gewoge der ganzen Spermienmasse.

\section{Kultivierung und Isolierung der bakteriellen Kulturen}

Zur Kultivierung und Isolierung benutzten wir das modifizierte Holst-Sturtevant-MediumHS Agar (Azuma und Kitaoka, 1965).

Von jeder Konserve wurden 2 Schalen mit festem Nährboden beimpft. Die eine Schale wurde aerob, die andere anaerob bebrütet. Die Bebrütung wurde bei $37^{\circ} \mathrm{C}$ bis zù 1 Woche durchgeführt.

Es wurden geklonte Subkulturen der angezüchteten Keime angelegt. Von diesen Subkulturen sind wir beim Feststellen der Eigenschaften der betreffenden Bakterien ausgegangen. Die Kulturen hielten wir auf Schrägagar bei $4^{\circ} \mathrm{C}$.

\section{Identifikation der angezüchteten Bakterien}

Zur Feststellung der biochemischen Eigenschaften und der anderen Bewertungskriterien benutzten wir eine Reihe von Testen.

Wir bewerteten Aussehen, Farbe und Größe der Kolonien, Sauerstoffbedürftigkeit, Größe und Anordnung der Mikroben im Präparat, Beweglichkeit, Gramreaktion, die Sporenfärbung nach Schaeffer-Fulton, das Anwachsen auf Fleischpepton-Bouillon, Ammoniakbildung, Harnstoffspaltung, Gelatinever flüssigung, Reduktion von Nitrat zu Nitrit, Schwefelwasserstoffbildung, ferner Fällung, Peptonisierung, Säuerung oder Alkalisierung von Milch, Stärkehydrolyse, die Methylrot-und die Voges-Proskauer-Reaktion, die Vergärung von Saccharose, das Wachstum auf Nährböden mit hoher Salzkonzentration, Katalase-und Oxydasereaktion.

Zu diesen Zwecken benutzten wir das Medium der sog. bunten Reihe nach ZaHRADNICKÝ und Kol. (1965).

\section{Desinfektion der Spritzennadel}

Zur sterilen Abnahme des Spermas haben wir die Spritzennadel durch Durchspülen mit $96 \%$ igem Äthylalkohol desinfiziert. Reste des Alkokols wurden mit destilliertem Wasser entfernt.

\section{ERGEBNISSE}

\section{a. - Mit konserviertem Sperma}

In Dol und in Liběchov stellten wir 1968 und 1969 insgesamt 50 Spermakonserven her. Bei allen Konserven haben wir die Motilität der Spermien geprüft, 33 Konserven untersuchten wir ausserdem bakteriologisch.

Die Ergebnisse der Motilitätsprüfungen sind in der Tabelle zusammengestellt. $\mathrm{Zu}$ grösseren Unterschieden in der Motilität der ohne und der mit Zusatz von Schutzstoffen konservierten Spermien kam es erst bei den 60 Tage gelagerten Konserven. Eine auffallend höhere Motilität wurde bei dem mit Zusatz von Chloramphenikol gelagerten Sperma festgestellt.

Die maximale Spermienbewegung war nach 3-30 Minuten zu beobachten, unabhängig von dem verwendeten Schutzstoff. Mit der Lagerungszeit verlängerte sich auch die zur Erreichung der maximalen Motilität benötigte Zeit.

Die Spermienbewegung dauerte bei allen Konserven bis zum Austrocknen 
des Tropfens - mit Ausnahme von drei 60 Tage lang gelagerten Konserven ( 1 mit Zusatz von Lysosym, 2 mit Zusatz von Lysosym und Chloramphenikol), bei denen die Bewegung auf nur einige Minuten beschränkt war.

Bakteriologisch wurden untersucht :

15 Spermakonserven ohne Zusatz eines Schutzstoffes. Alle diese Konserven hatten beim Öffnen ein normales Aussehen und waren ohne üblen Geruch. Vom Inhalt von 5 Konserven wurden nach dem Öffnen folgende Bakterien angezüchtet, isoliert und identifiziert : Streptococcus epidermidis, Bacillus brevis; in einem Falle wurden aerobe Stäbchen der Familie Bacillaceae gefunden; es gelang nicht, sie näher zu bestimmen. Der Inhalt der anderen Konserven war steril.

12 Spermakonserven mit Zusatz von Lysosymlösung in den Konzentrationen 1, 5 und $10 \mathrm{mg} / \mathrm{ml}$. Alle Konserven hatten beim Öffnen ein normales Aussehen und waren ebenfalls ohne üblen Geruch. Von dem Inhalt von 3 Konserven wurden folgende Bakterien angezüchtet, isoliert und identifiziert : Gaffkya tetragena, Citrobacter (Escherichia) freundii; in einem Falle wurden aerobe Stäbchen der Familie Bacillaceae gefunden; es gelang nicht, sie näher zu bestimmen. Zwischen den einzelnen Konzentrationen der Lysosymlösung $(1,5$ und $10 \mathrm{mg} / \mathrm{ml})$ wurden keine Unterschiede gefunden.

2 Spermakonserven mit Zusatz von Chloramphenikollösung in der Konzentration $1 \mathrm{mg} / \mathrm{ml}$. Beide Konserven hatten beim Öffnen ein normales Aussehen und waren ohne Geruch. Der Inhalt war steril.

4. Spermakonserven mit Zusatz von Chloramphenikol- und Lysosymlösung in der Konzentration $1+1 \mathrm{mg} / \mathrm{ml}$. Alle Konserven hatten beim Öffnen ein normales Aussehen und waren ohne Geruch. Der Inhalt erwies sich als steril.

\section{b. - Mit frischem Sperma}

Bei 40 Drohnen haben wir Sperma und Schleim unmittelbar von den Begattungsorganen abgenommen und je einen Sperma- und Schleimtropfen auf HS-Agar ausgebreitet. Die Kultur war in allen Fällen negativ.

Ferner haben wir in 10 Fällen Sperma kultiviert, das mit einer nicht desinfizierten Nadel abgenommen worden war. Nach 24. Stunden wurde auf allen Schalen ein starker Bakterienbewuchs festgestellt. Diese Bakterien wurden mikroskopisch als grampositive Kokken identifiziert. Dagegen waren alle mit einer desinfizierten Nadel durchgeführten Abnahmen steril.

\section{DISKUSSION}

Die Motilität der Spermien halten wir für ein geeignetes Kriterium für die Verwendbarkeit des konservierten Spermas. Bei Probebesamungen zeigte 
sich, daß unbewegliches Sperma (Motilitätsstufen 0 und 1) nicht mehr in die Samenblasen der Königinnen gelangt. Als unterste Grenze, bei der Besamungen wenigstens teilweise erfolgreich sind, kann man die Motilitätsstufe 2 ansehen.

Aus der Tabelle ist ersichtlich, daß bei 60-tägiger Lagerung nur Konserven mit Zusatz von Chloramphenikol und Lysosym mit Chloramphenikol diese Grenze überschritten haben.

Das frische Sperma war in unseren Versuchen immer steril. Den Befund polnischer Autoren (Galuschкo, Niemczuk und Tomaschewska, 1965), die in unmittelbar in die Spritzennadel des Besamungsapparates aufgesaugtem und sofort kultiviertem Sperma Streptococcus apis feststellten, konnten wir nicht bestätigen.

Die Infektion des Spermas erfolgt sekundär bei der Abnahme des Spermas, bei der Herstellung und eventuell auch beim Öffnen der Konserven. Der positive Befund bei den lysosymierten Konserven wurde höchstwahrscheinlich durch Infektion beim Öffnen verursacht, weil es sich um die ersten Konserven handelte, bei denen wir die Öffnungsmethode noch nicht beherrschten. Dies zeigt auch der Umstand, daß gerade Gaffkya tetragena isoliert wurde, das gegen Lysosym sehr empfindlich ist.

Alle im Sperma gefundenen Mikroorganismen gehören zur banalen apathogenen Mikroflora. Sterilität kann ebenso gut durch eine sterile Abnahme des Spermas wie durch den Zusatz von Lysosym oder Chloramphenikol erreicht werden.

Durch Gewährleistung der Sterilität verhindert man die vorzeitige Entwertung des Konserveninhalts. Dennoch wird die maximale Lagerungsfrist nicht verlängert. Es scheint, daß manche Schutzstoffe (Chloramphenikol) eine günstige Nebenwirkung auf die Erhaltung der Vitalität der Spermien ausüben, weil sie in den Metabolismus derselben eingreifen. Chloramphenikol ist durch seine Hemmwirkung auf die Proteinsynthese und teilweise auch auf die DNS-Synthese der Bakterien bekannt (GaLE, 1963).

Weitere Bemühungen, die Lagerungsfähigkeit des Bienenspermas zu erhöhen, könnten gerade in dieser Richtung geführt werden - zur biochemischen Begrenzung des Spermien-Metabolismus. Es ist nicht ausgeschlossen, daß auch niedrige Temperaturen günstig wären. Die bisherigen Erkenntnisse über die Wirkung niedriger Temperaturen weichen stark von einander ab (Taber, 1961, Lensky und Schindler, 1967, Savada und Chang, 1964). In dieser Hinsicht wären biochemische Untersuchungen der Spermathekaldrüse sehr nützlich; denn diese Drüse spielt eine wichtige Rolle bei der Aufbewahrung des Spermas in der Samenblase der Bienenkönigin (Koeniger, 1969). 


\section{RÉSUMÉ}

Bien que les spermatozoïdes d'abeille restent en vie plusieurs années in vivo, c'est-à-dire dans la spermathèque de la reine, on n'a jamais pu résoudre jusqu'ici de façon satisfaisante le problème de leur conservation in vitro.

'́aber et Blum (1960) et šKnobaL, VeselÝ et HrdÝ (1966) ont tronvé fréquemment dans le sperme conservé des signes très nets d'infection. Ils voient dans l'infection hactérienne l'une des cause possibles de la courte durée de vie du sperme conservé.

C'est pourquoi le but du présent travail est de déterminer dans quelle mesure on peut agir sur la motilité et par conséquent sur la qualité du sperme par une conservation stérile.

Le sperme conservé a été obtenu par la méthode de TABER (1961). Le sperme pur, sans mucus est aspiré sur les organes d'accouplement éversés de mâles âgés de plus de 12 jours provenant de reines fécondées et non fécondées appartenant à la race carnica. L'éversion est obtenue par anesthésie des mâles aux vapeurs de chloroforme.

Pour la protection du sperme contre les infections bactériennes nous avons utilisé le lysozyme en solution concentrée. Nous avons préparé des solutions à 10,5 et $1 \mathrm{mg} / \mathrm{ml}$. Les solutions protectrices ont été ajoutées au prélèvement de sperme dans la proportion constante de 1 à 10 .

Nous avons éprouvé la motilité en goutte pendante avec $0,04 \mathrm{ml}$ d'une solution de $\mathrm{Na} \mathrm{Cl}$ à $1 \%$. La motilité maximum a été appréciée selon l'échelle suivante :
$0=$ spermatozoïdes sans mouvements, ni structure.
$1=$ spermatozoïdes sans mouvements perceptibles.
$2=$ mouvements lents de spermatozoïdes isolés.
$3=$ mouvements sur place de petits groupes de spermatozoïdes.
$4=$ mouvements sur place de la plupart des spermatozoïdes.
$5=$ mouvements spirales de grands paquets de spermatozoïdes.
$6=$ mouvements spirales violents de toute la masse de sperme.

La mise en culture a été effectuée en partie en conditions aérobies, en partie en conditions anaérobies.

Au total 50 échantillons de sperme conservé ont été préparés dont 33 ont été examinés du point de vue bactériologique. Parallèlement, du sperme frais provenant directement des organes sexuels a été mis en culture.

Nos résultats permettent de tirer les conclusions suivantes :

$1^{\circ}$ Le sperme, primitivement stérile est infecté secondairement par le prélèvement sur les organes du mâle, par la préparation ou par l'ouverture de la conserve.

$2^{\circ}$ Les bactéries contenues dans le sperme infecté ont été identifiées comme étant Streptococcus eperdermidis, Bacillus brevis, Gaffkya Tetragena et Citrobacter (Escherichia) freundii. Toutes les espèces trouvées appartiennent à la micro flore banale et, dans aucun cas il ne s'agissait de formes pathogènes pour l'abeille ou pour l'homme. La source d'infection la plus courante était la peau de l'homme.

$3^{\circ}$ On peut garantir la stérilité de la conserve par un prélèvement soigneux du sperme ou addition de Lysozyme ou de chloramphénicole en solution à la concentration de $1 \mathrm{mg} / \mathrm{ml}$ dans la proportion de $1: 10$ de sperme.

$4^{\circ}$ La stérilité protège le sperme conservé d'une dégradation prématurée mais ne prolonge pas la durée maximale de stockage (60 jours).

$5^{\circ}$ Avec le chloramphénicole on a découvert une action secondaire jusqu'ici inconnue : la motilité des spermatozoïdes conservés pendant 60 jours est notablement augmentée.

$6^{\circ}$ De nouvelles recherches devraient être faites sur cette action secondaire au chloramphénicole ou d'autres substances agissant sur le métabolisme des spermatozoïdes. 


\section{LITERATUR}

AzUma K., Kitaoka S., 1965 : Cultivation and properties of Bacillus larvae and decrease o. its proteolytic activity on glucose media. Nat. Inst. Anim. Hlth. Quart. 5 (3) : 138-145.

GALE E. F., 1963 : Mechanism of actions antibiotics. Pharmacological Reviews 15 (3) : $481-530$.

Galuschko H., Niemczuk R., Tomaschewska B., 1965 : Mikrobiologische Studien über das Sperma der Drohnen. Ref. — Der XX. internationale Jubiläumskongress der Bienenzüchter, Bucharest.

Koeniger Gudrun, 1969 : Experiments concerning the physiology of the spermatheca of the queen bee (Apis mellifica L.). Ref. - XXI. Congress of Apimondia in Munich.

LENSKY D., Schindeer H., 1967 : Motility and reversible insactivation of honeybee spermatozoa in vivo and in vitro. Annales de l'abeille 10 (1) : 5-16.

Modr Z., Heřmanský M., Vlček V., 1969 : Antibiotika a jejich lékové formy. Praha.

Poole H. K., Taber St., 1969. A method of in vitro storage of honey-bee semen. Am. Bee J. 109 (11), 420-421.

Savada J., Chang M. C., 1964 : Tolerance of honey bee sperm to deep freezing. Journal of Economic Entomology 57 (6) : 891-892.

SEdLÁK J. et al., 1962. Repetitorium lékařské mikrobiologie. Praha.

Stárka J., 1959 : Fysiologie a biochemie mikrobu. Praha.

šKrobal D., VeselY V., HrdÝ I., 1966 : Einleitung zur Diskussion über die Probleme der Drohnen-Sperma-Konservierung. Ref. - Internationales Symposium der Apimondia, Zvíkovské Podhradí.

TABER ST., 1961 : Successful shipment of honeybee semen. Bee World 42 (7) : 173-176.

Taker St., Brum M. S., 1960: Preservation of honey bee semen. Science $131: 1734-1735$.

VESELÝ V., 1961 : Př́íspěvek $k$ otázce umělého osemeňování včelích matek. Zoologické listy $\mathrm{X}(3): 203-210$.

ZamradnickÝ J. und Kol.,"1956 : Speciální mikrobiologie. Praha. 